\title{
Schnelles drahtloses Funknetzwerk mit energieautarken Kommunikationsknoten
}

M.Sc. Rainer Hornung

Professur für Elektrische Messtechnik Helmut-Schmidt-Universität, Universität der Bundeswehr, Hamburg

Holstenhofweg 85, 22043 Hamburg

E-Mail: rainer.hornung@hsu-hh.de

Tel.: +494065412939

\author{
Dr.-Ing. Ralf Heynicke \\ Professur für Elektrische Messtechnik \\ Helmut-Schmidt-Universität, Universität \\ der Bundeswehr, Hamburg \\ Holstenhofweg 85, 22043 Hamburg \\ E-Mail: ralf.heynicke@hsu-hh.de \\ Tel.: +494065412920
}

\author{
Univ.-Prof. Dr.-Ing. Gerd Scholl \\ Professur für Elektrische Messtechnik \\ Helmut-Schmidt-Universität, Universität \\ der Bundeswehr, Hamburg \\ Holstenhofweg 85, 22043 Hamburg \\ E-Mail: gerd.scholl@hsu-hh.de \\ Tel.: +494065413341
}

\section{Kurzfassung:}

In diesem Beitrag wird ein drahtloses Kommunikationssystem vorgestellt, dass die Anforderungen der Fertigungsautomatisierung in Bezug auf Latenzzeit und Paketfehlerrate erfüllt und dessen abgesetzte Sensormodule aus der Energie der Raumbeleuchtung gespeist werden. Es wird erläutert wie durch eine geeignete Auslegung der Basisstations-Hardware und des Kanalzugriffs sowie durch unterschiedliche Betriebsmodi der abgesetzten Kommunikationsknoten ein energieautarker Betrieb realisiert werden kann. Dies konnte in ersten Messungen anhand eines Demonstratorsystem bei einer Auslastung von zwei Ereignissen pro Sensor und Sekunde unter Einhaltung der Latenzzeiten von weniger als $10 \mathrm{~ms}$ bei einem Netzwerk mit 400 Kommunikationsknoten belegt werden.

\section{Einleitung:}

Drahtlose Technologien haben in der Zwischenzeit, auch mit Blick auf ihre Zuverlässigkeit, einen hohen Reifegrad erreicht und haben sich infolge dessen in industriellen Umgebungen etabliert. Bisher wurden drahtlose Netzwerke, wie z.B. WirelessHART, vor allem in der Prozessautomatisierung eingesetzt, um abgesetzte Sensoren in relativ großen Zeitabständen abzufragen [1]. Drahtlose Technologien können allerdings erst dann ihr volles Potential entfalten, wenn auch die Energie zum Betrieb des Sensornetzwerkes drahtlos zur Verfügung gestellt wird. Aufgrund des relativ geringen Kommunikations- und damit Energiebedarfs lässt sich diese Forderung im Bereich der Prozessautomatisierung bereits häufig erfüllen. Im Bereich der Fertigungsautomatisierung sind die geforderten Latenzzeiten in der Regel erheblich kürzer. Beispielsweise wurde in den öffentlich geförderten Projekten ENAS und MIKOA Latenzzeiten von unter $10 \mathrm{~ms}$ für die Datenerfassung und Datenübertragung vom Sensor bis zur Umsetzung in der SPS gefordert. Dabei darf gleichzeitig die Restfehlerwahrscheinlichkeit des Systems, hier abgekürzt durch SER (System Error Rate), von $10^{-9}$ nicht überschritten werden. Weiter wurde eine maximal auftretende mittlere Ereignisrate von 2 Ereignissen pro Sensor und Sekunde gefordert. Am Markt befindliche drahtlose energieautarke Kommunikationsknoten nutzen meist Trägerfrequenzen bei $433 \mathrm{MHz}$ bzw. $868 \mathrm{MHz}$, da für diese Frequenzbänder Standard-Funktransceiver erhältlich sind, die einen relativ geringen Energiebedarf im Sende- und Empfangsbetrieb aufweisen. Da diese Systeme relativ schmalbandig ausgelegt sind, ist die mögliche Übertragungsrate vergleichsweise gering [2]. Im Gegensatz hierzu verfügen HFTransceiver im 2,4 GHz ISM-Band aufgrund der größeren erlaubten Bandbreite über eine höhere Datenübertragungsrate. Beispielsweise steht mit IEEE 802.15.1 ein Funkstandard bzw. eine Übertragungstechnik zur Verfügung, die Übertragungsraten von bis zu $1 \mathrm{MBit} / \mathrm{s}$ ermöglicht [3]. Der Energiebedarf derartiger HF-Transceiver ist bei gleicher übertragenen Datenmenge mit den Transceiver, die in den niederfrequenten Frequenzbereichen betrieben werden, durchaus vergleichbar.

\section{Systembeschreibung:}

Die Umgebung in der Fertigungsautomatisierung ist meist durch metallische Oberflächen unterschiedlichster Strukturen geprägt. Dies hat bei Funkübertragungen ausgeprägte Mehrwegeausbreitung zur Folge, die insbesondere ohne direkte Sichtverbindung einen stark frequenzselektiven und zeitvarianten Funkkanal erzeugt. Daneben wird die Auslastung der Funkkanäle im 2,4 GHz-ISM-Band auch in der Fertigungsautomatisierung durch die stetig steigende Anzahl an drahtlosen Systemen wie z.B. WLAN und Bluetooth immer höher. Die Mehrwegeausbreitung und die steigende Auslastung der Funkkanäle können die Anzahl an notwendigen Wiederholungen bis zu einer Übertragung eines Datenpaketes mit einer $S E R<10^{-9}$ und 
damit die Latenzzeit entscheidend erhöhen. In diesem Beitrag wird daher beschrieben, wie durch ein angepasstes CSMA (Carrier Sense Multiple Access)-Protokoll, bei dem die abgesetzten Sensor/Aktorknoten mehr oder weniger spontan auf den Funkkanal zugreifen, ein Echtzeit-System mit minimalem Management- und Energieaufwand und gleichzeitig großer Zuverlässigkeit realisiert werden kann.

\subsection{Hardware-Architektur:}

Der prinzipielle Aufbau von drahtlosen Sensor/Aktor- Kommunikationssystemen für die Fertigungsautomatisierung ist in Abbildung 1 dargestellt. Eine zentrale Basisstation (BS) ist als Gateway einerseits über ein Feldbussystem an eine speicherprogrammierbare Steuerung (SPS) angeschlossen und kommuniziert andererseits über Funk mit abgesetzten Kommunikationsknoten [4].

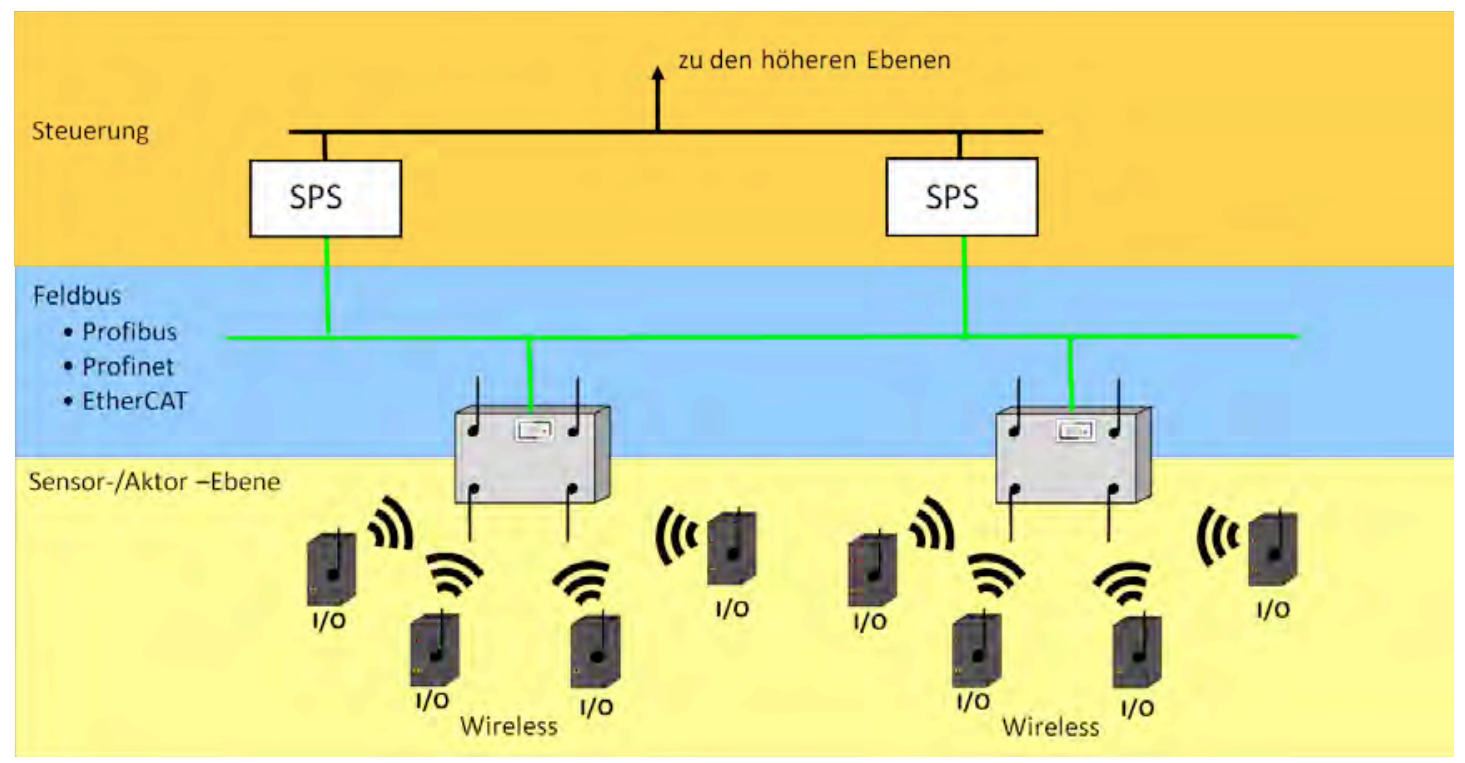

Abbildung 1: Prinzipieller Aufbau eines drahtlosen Sensor/Aktor- Kommunikationssystems.

Aufgrund der geforderten geringen Latenzzeiten und Fehlerraten erfolgt der Medienzugriff bei den meisten am Markt befindlichen Systemen über ein F/TDMA-Verfahren. Die für die Kommunikation zur Verfügung stehenden Zeit- und Frequenzschlitze werden dabei in der Regel in einem Konfigurationsmodus den Sensor/Aktor-Knoten fest zugeordnet. Diese feste Zuordnung hat den Vorteil, dass die Kommunikation streng determiniert abläuft, benötigt aber relativ viel Energie in den abgesetzten Kommunikationsmodulen, um die notwendige zeitliche Synchronisation zwischen ihnen und der BS aufrecht zu erhalten [5].

In Abbildung 2 ist auf der linken Seite ein Blockschaltbild der BS des Testsystems dargestellt. Mit Hilfe eines FPGAs können die angeschlossenen HF-Transceiver parallel angesteuert werden. Im ARM9- $\mu$ Controller ist die Anwendungsschicht des Protokoll-Stacks implementiert sowie die Anbindung der BS an das übergeordnete Feldbussystem über ein Standard-Feldbusinterface. Auf der rechten Seite ist ein Bild der BS zu sehen. Das System ist redundant ausgelegt, so dass gleichzeitig zehn HF-Transceiver auf der BS angesteuert werden können. Davon werden acht für den Uplink und zwei für Downlink genutzt. 

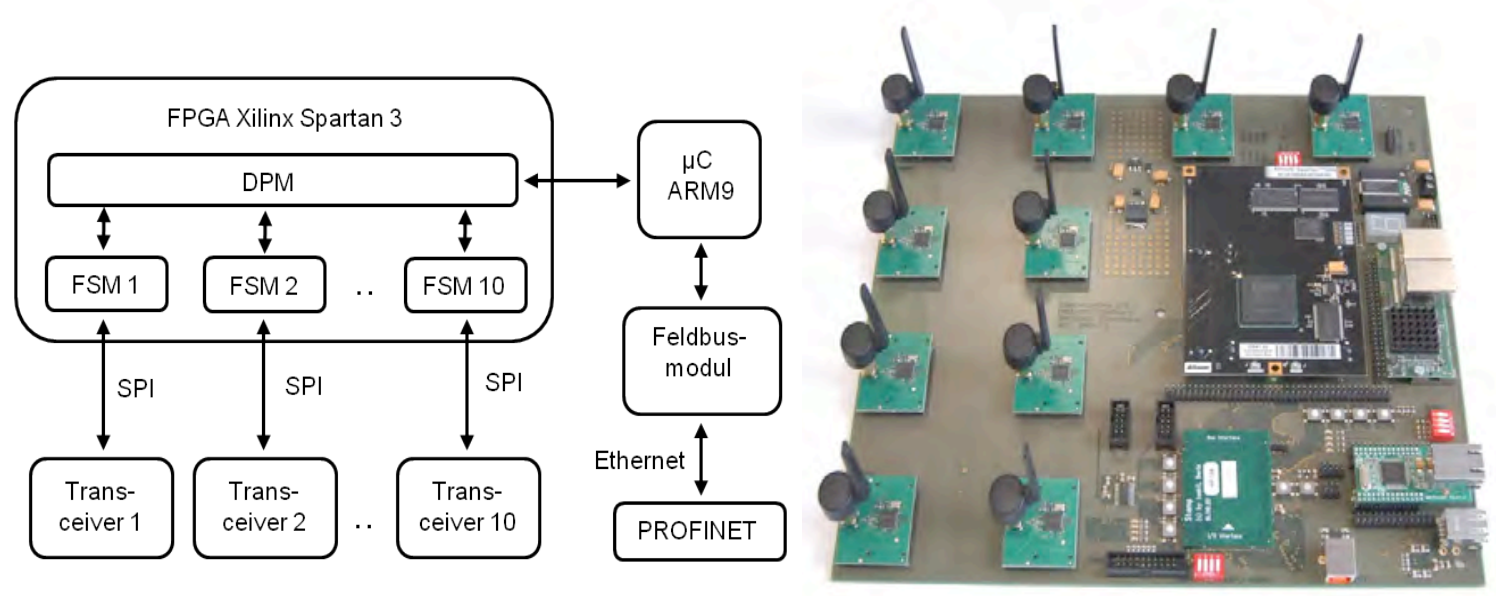

Abbildung 2: Blockschaltbild (links) und Demonstrator-Aufbau (rechts) der Basisstation.

Für den energieautarken Betrieb der Sensor-Kommunikationsmodule müssen diese über Energie-Harvester Energie aus der Umgebung „ernten“. Untersuchungen [6] von EnergieHarvestern haben deutlich gemacht, dass aus einem kleinen Volumen in der Regel auch nur wenig Energie generiert werden kann und die Energieerzeugung immer sehr spezifisch auf die jeweilige Anwendung hin zugeschnitten werden muss. Im Gegensatz hierzu bietet eine StandardSolarzelle eine vergleichsweise einfache Möglichkeit, platzsparend Energie zu „ernten“, da in den meisten Industriezweigen gemäß DIN EN 12464 [7] eine Beleuchtungsstärke zwischen $E_{V}=500 \mathrm{~lx}$ und $E_{V}=1500 \mathrm{~lx}$ vorschreiben ist, und deshalb in der Regel mit der Beleuchtung ausreichend Energie zur Verfügung steht.

Bei dem realisierten System wurde ein Solarmodul der Größe $92 \times 66 \mathrm{~mm}$ eingesetzt. Bei einer Beleuchtungsstärke von $E_{V}=500 \mathrm{~lx}$ weist dieses im Maximum Power Point eine Spannung von $U_{\text {MPP }}=1,4 \mathrm{~V}$ und eine Stromstärke von $I_{\text {MPP }}=516 \mu \mathrm{A}$ auf. Der Step-Up DC/DC-Converter LTC3105 [8] generiert aus der von der Solarzelle zur Verfügung gestellten Spannung eine maximale Spannung von $U_{D C D C}=4,2 \mathrm{~V}$. Als Energiespeicher wurde ein Li-lonen- Akku mit einer Nennspannung von $U_{\text {Li-lonen }}=3,7 \mathrm{~V}$ und einer Kapazität von $Q=100 \mathrm{mAh}$ eingesetzt. Der $\mu$ Controller zur Steuerung des HF-Transceivers ist ein 16 Bit Ultra- Low-Power RISC Prozessor vom Typ MSP430F1612. Dieser verfügt über besonders energieeffiziente Sleep-Modi und bietet gleichzeitig die Möglichkeit, die Sensorinformation als Interrupt-Signal zu nutzen [9]. Als HFTransceiver wird ein TI/Chipcon CC2400 [10] eingesetzt.
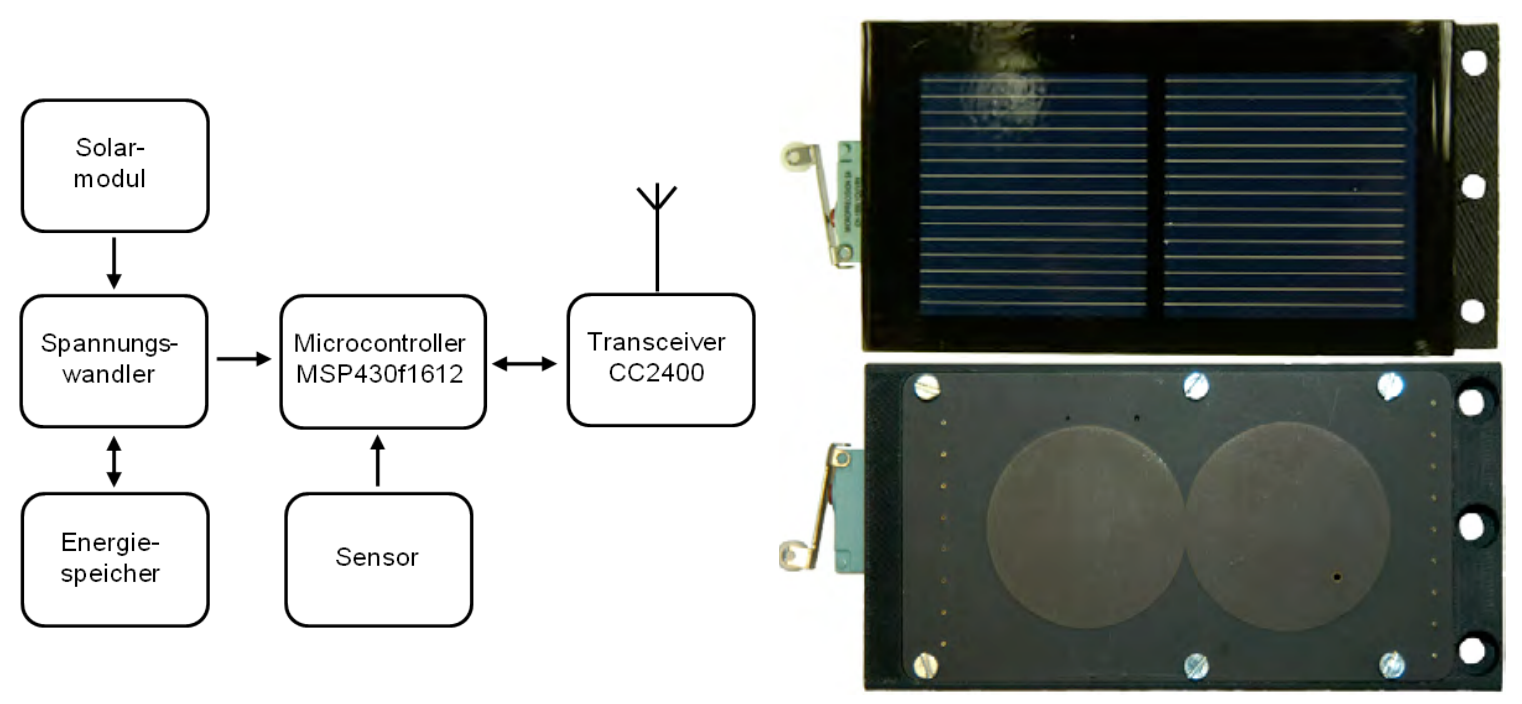

Abbildung 3: Prinzipieller Aufbau (links) und Realisierung eines energieautarken drahtlosen Endschalters (rechts). 
In Abbildung 3 ist links der Aufbau des Sensor-Kommunikationsmoduls skizziert, auf der rechten Seite ist ein Foto der Oberseite mit der aufgebrachten Solarzelle und ein Bild der Unterseite mit der integrierten Antenne zu sehen. Am linken Rand der Bilder ist jeweils ein Endschalter zu sehen.

Bei ersten Untersuchungen des Energiehaushaltes des Kommunikationsknoten wurde bei einer Beleuchtungsstärke von $E_{V}=500 \mathrm{~lx}$ der in Abbildung 4 dargestellte Ladestrom des Li-lonenAkkus über einen Kommunikationszyklus gemessen. Der Ladestrom ist stark vom aktuellen Ladezustand des Akkus abhängig und schwankt zwischen $I_{L}=2 \mu \mathrm{A}-30 \mu \mathrm{A}$. In der Ruhephase beträgt der mittlere Ladestrom ca. $I_{L}=20 \mu \mathrm{A}$. In der Sende/Empfangsphase wurde ein mittlerer Pufferstrom von ca. I $=-5,5 \mathrm{~mA}$ gemessen.

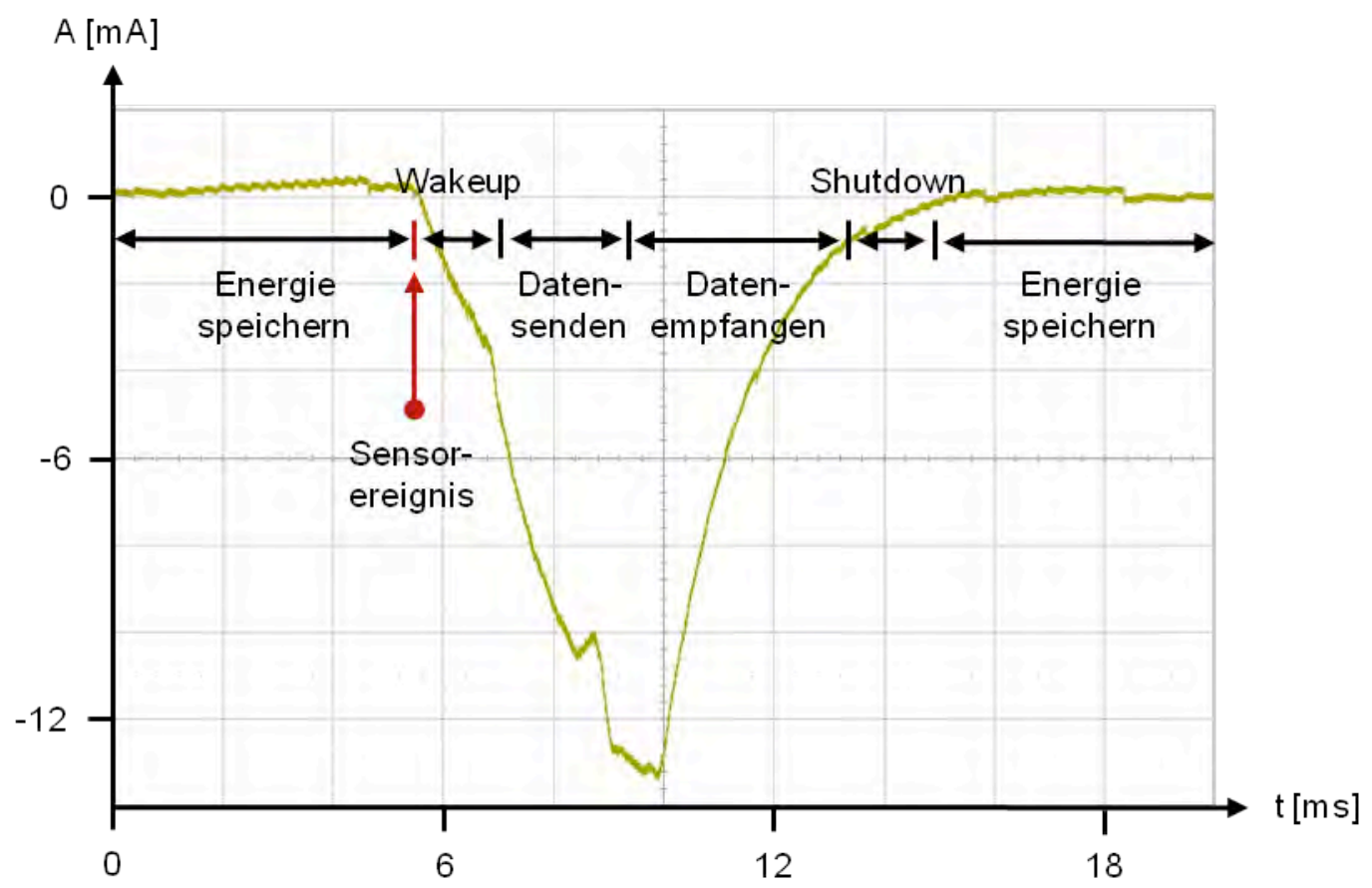

Abbildung 4: Ladestrom des im Sensor-Kommunikationsknoten integrierten Li-lonen-Akkus über einen Kommunikationszyklus.

3.2. Medienzugriff:

Eigene Messungen der Funkkanäle in industriellen Umgebungen haben gezeigt, dass dort ein Großteil der Funkkanäle weitgehend zeitinvariant ist. In Abbildung 5 ist beispielhaft das Spektrogramm einer gemessenen Übertragungsfunktion dargestellt. Deutlich zu sehen sind „Frequenzen“ mit einer geringen Pfaddämpfung (rot) und „Frequenzen“ mit einer relativ hohen Pfaddämpfung (blau) [11].

Für die Auswahl von Frequenzkanälen mit einer möglichst geringen Pfaddämpfung wird innerhalb einer Startup-Phase die Übertragungsfunktion $H_{\mathrm{ik}}(f)$ zwischen dem i-ten HF-Transceiver der BS und Knoten $k$ über das gesamte 2,4 GHz-ISM-Band vermessen, d.h. der RSSI-Wert der Knoten ausgelesen [12]. Aus diesen Messungen werden dann für alle Kommunikationsknoten zwei Downlink-Kanäle und pro Kommunikationsknoten vier Uplink-Kanäle ausgewählt. Jeder Kommunikationsknoten wird daher von einem anderen Satz von HF-Transceiver auf der Basisstation bedient. Bei der Auswahl der Frequenzkanäle wird eine möglichst gleichmäßige Auslastung der HF-Transceiver auf der BS angestrebt, so dass eine minimale Latenzzeit garantiert werden kann. Die in der Startup-Phase bestimmten Frequenzkanäle werden in Tabellen zusammengefasst und am Ende der Startup-Phase an jeden Sensor/Aktor-Knoten übertragen. Anschließend geht das Funksystem in den normalen Betrieb über [13] [14]. 


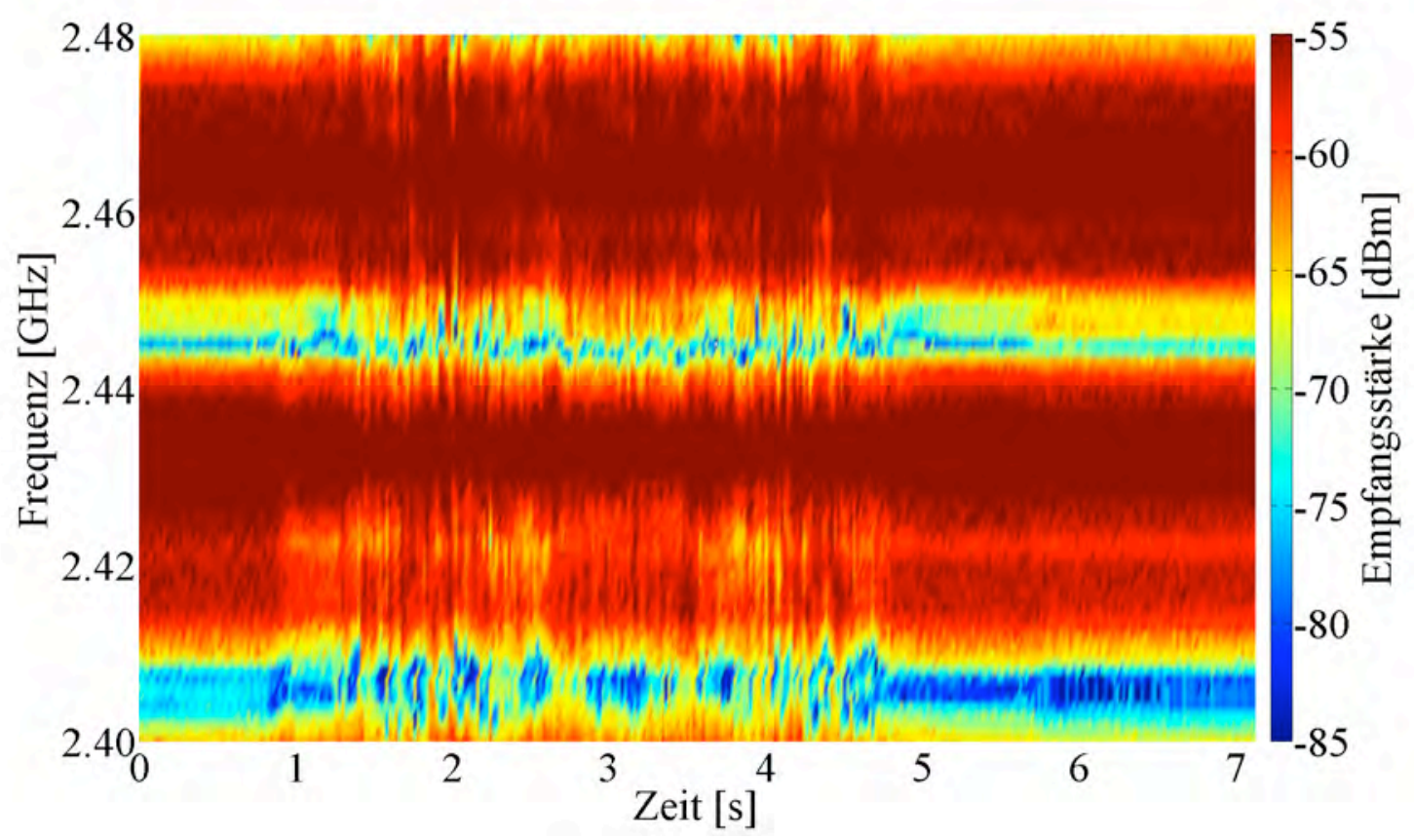

Abbildung 5: Spektrogramm eines gemessenen Funkkanals.

Zur Steigerung der Koexistenzfähigkeit mit im 2,4 GHz ISM-Band gleichzeitig betriebenen Funksystemen können in der Startup-Phase gezielt Frequenzkanäle ausgeblendet werden.

Bereits in den siebziger Jahren wurden verschiedene Verfahren für einen unsynchronisierten Medienzugriff (MAC) untersucht und erstmalig unter dem Synonym ALOHA [15] vorgestellt. Grundlage für die Berechnungen der Kanalbelegung bildete hierbei ein Poisson-Prozess für die Einspeisung der Kommunikationspakete in den Kanal.

Ist $T_{\mathrm{p}}$ die Länge eines Kommunikationspaketes, dann lässt sich unter der Annahme eines Poisson-Prozesses die Wahrscheinlichkeit berechnen, dass es zu keiner Kollision kommt. Diese ist durch

$$
P=e^{-\frac{T_{P}}{\Delta \tau}}
$$

gegeben, wobei $\overline{\Delta t}$ der mittlere Abstand zwischen zwei Paketen ist. Unter dem Schlagwort CSMA (Carrier Sense Multiple Access) wurde dieses Verfahren weiterentwickelt.

CSMA-Verfahren überprüfen den aktuellen Status des zu nutzenden Funkkanals, um mögliche Kollisionen zu vermeiden. Dieselbe Technik wird in aktuellen Systemen unter dem Begriff Listen Before Talk (LBT) verwendet. Das LBT-Verfahren konnte auf der unter 3.1 beschriebenen Hardware sehr effizient umgesetzt werden, da der zeitliche Abstand zwischen der Überprüfung des Funkkanals und dem Absetzen eines Datenpaketes auf nur. $t_{\mathrm{a}}=25 \mu \mathrm{s}$ reduziert werden konnte. Der notwendige, auf die Paketlänge $T_{\mathrm{p}}$ normierte Durchsatz $S$ eines Funksystems berechnet sich aus der mittleren Ereignisrate $\bar{E}$ pro Kommunikationsknoten und der Anzahl der Kommunikationsknoten $\mathrm{N}$ zu

$$
S=E \cdot N \cdot T_{P}
$$

Dies entspricht bei einer mittleren Ereignisrate von 2 Datenpaketen pro Sekunde, einer Paketlänge von $T_{\mathrm{p}}=128 \mu \mathrm{s}$ und $\mathrm{N}=50$ Sensorknoten pro Frequenzspur einen mittleren zeitlichen Abstand von $\overline{\Delta t}=10 \mathrm{~ms}$ zwischen den einzelnen Paketen. Gemäß [16] besteht zwischen dem Durchsatz $S$ und der Angebotsrate $G$ der Zusammenhang

$$
S=\frac{G \cdot e^{-a \cdot G}}{G \cdot(1+2 a)+e^{-a \cdot G}} .
$$


Der Parameter a steht hierbei für den Quotienten aus dem zeitlichen Abstand $t_{\mathrm{a}}$ und der Paketlänge $T_{\mathrm{P}}$. In unserem Fall setzt sich das Datenpaket zusammen aus vier Byte Präambel, vier Byte Sync-Word, sechs Byte Daten sowie zwei Byte Cyclic Redundancy Check (CRC). Damit berechnet sich die Länge des Datenpaketes zu $T_{\mathrm{P}}=128 \mu \mathrm{s}$. Damit ist $a=0,2$, was mit $S=0,0128$ einem $G=0,013$ entspricht, daraus folgt ein mittlerer Paketabstand von 9,8 ms. Der in Gleichung (3) formulierte Zusammenhang ist in Abbildung 6 graphisch dargestellt.
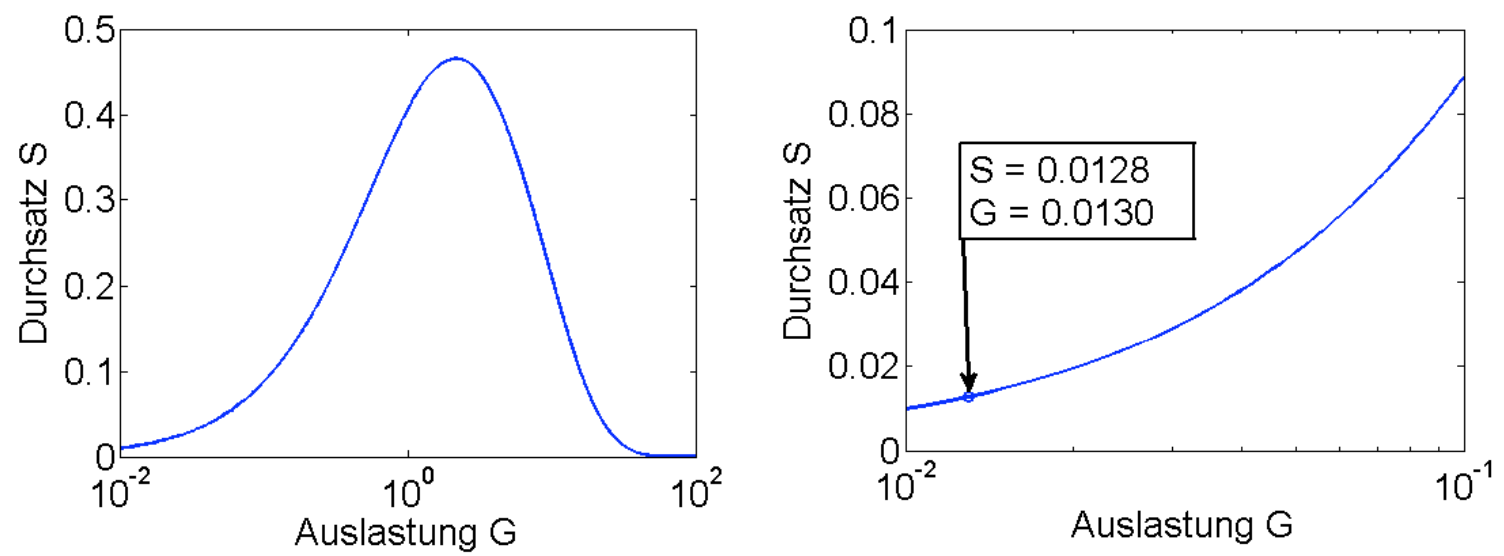

Abbildung 6: Darstellung des Durchsatzes S des realisierten nonpersistent CSMA-Verfahren über der Auslastung G.

In der Fertigungsautomatisierungstechnik ist die maximal auftretende Latenzzeit $t_{L}<10 \mathrm{~ms}$ bei einer Restfehlerwahrscheinlichkeit des Systems von $S E R<10^{-9}$ für das gesamte Netzwerk von großer Bedeutung. Um eine derart geringe Restfehlerwahrscheinlichkeit erreichen bzw. unterschreiten zu können, sind Wiederholungen der Datentelegramme unumgänglich. Die SER lässt über

$$
\begin{aligned}
& S E R>\left(1-\frac{S_{\max }}{G_{\max }} \cdot(1-P E R)\right)^{n \max } \\
= & \left(1-\frac{S \cdot(1+\bar{n})}{G_{\max }} \cdot(1-P E R)\right)^{n} \max
\end{aligned}
$$

bestimmen. Hierin bedeuten PER die Packet Error Rate der eingesetzten HF-Transceiver, $\bar{n}$ die mittlere Anzahl der notwendigen Wiederholungen, $S_{\max }$ und $G_{\max }$ Durchsatz und Angebotsrate unter Berücksichtigung der mittleren Anzahl der Wiederholungen. $n_{\max }$ kennzeichnet die maximale Anzahl notwendiger Wiederholungen für das Erreichen der vorgegebenen SER. Für den CC2400 wurde in der Testumgebung eine mittlere Paketfehlerrate von PER = 0,01 bestimmt. Die mittlere Anzahl an Wiederholungen beträgt $\bar{n}=0,010203$.

Die für eine zu unterschreitenden Restfehlerwahrscheinlichkeit von $S E R<10^{-9}$ maximal notwendige Wiederholungsanzahl $n_{\max }$ kann durch Umformung der Gleichung (4) berechnet werden. Sie beträgt $n_{\max }=4,9$ und wird auf $n_{\max }=5$ Wiederholungen gerundet.

Um bei Kollisionen den zeitlich spontan auftretenden Datenstau schnell beheben zu können, wurde eine zusätzliche Routine zur Reduzierung der Kollisionswahrscheinlichkeit im MACProtokoll implementiert. In dieser Routine wird der Sendezeitpunkt um ein Vielfaches des zeitlichen Abstands $t_{a}$ über eine Zufallszahl, die im Intervall zwischen 1 und 8 liegt, variiert. Die Wahrscheinlichkeit dafür, dass zwei Ereignisse innerhalb des Zeitabstandes $t_{a}=25 \mu s$ auftreten, beträgt bei der beschriebenen Poisson-Verteilung $10^{-5,5}$. Mit der Routine zur Reduzierung der Kollisionswahrscheinlichkeit kann diese innerhalb von zwei Durchläufen auf ca. $10^{-9,5}$ gesenkt werden, so dass die geforderte SER von $10^{-9}$ mit einem relativ einfachen CSMA-Verfahren erreicht werden kann. 


\subsection{Protokollaufbau:}

Bei dem hier untersuchten Kommunikationssystem werden den Funkteilnehmern keinen Zeit- und Frequenzschlitze zugeteilt. Die Kommunikation mit der BS verläuft komplett asynchron, d.h. die Basisstation und die Kommunikationsknoten senden ihre Daten nur wenn die Aktoren angesteuert werden müssen oder ein Sensorereignis am Kommunikationsmodul anliegt. Bei Bedarf kann zusätzlich sporadisch ein Alive-Signal abgesetzt werden. Wird vom Sensor ein Zustandswechsel an das Kommunikationsmoul gemeldet, wechselt dieses aus dem Energiesparmodus in den aktiven Sende/Empfangsmodus und setzt nach Überprüfung des Kanalstatus unmittelbar seine Daten an die BS ab (siehe Abbildung 4). Nach Quittierung (ACK) durch die BS geht der Kommunikationsknoten wieder in den Energiesparemodus. Es gibt zwei verschiedene ACK-Typen: ein einfaches Acknowledgement (ACK1) und eine Variante, bei der gleichzeitig mehrere Sensor/Aktor-Module adressiert werden (ACK2). ACK1 enthält nur eine Identifikationsmarke (ID) für einen bestimmten Kommunikationsknoten, ACK2 kann bis zu 20 IDs enthalten. Nachdem die BS die Daten eines Kommunikationsknotens empfangen hat und die Daten auf ihre Korrektheit überprüft wurden setzt die BS ACK1 nach einer Verzugszeit von $t_{A C K}=500 \mu \mathrm{s}$ ab. Empfängt die BS mehrere Kommunikationsknoten innerhalb eines Zeitfensters von $t_{W}=250 \mu$ s auf einer oder mehreren Uplink-Spuren, wird ACK2 ebenfalls nach einer Verzugszeit von $t_{A C K}=500 \mu s$ an alle betreffenden Teilnehmer verschickt. Die Zeitspanne zwischen Begin eines Uplink-Pakets und dessen Quittierung über ein Downlink-Paket wird hier als Framedauer $t_{\mathrm{F}}$ bezeichnet. Die Framedauer beträgt $720 \mu \mathrm{s}$. Erhält der Kommunikationsknoten kein ACK, dann wechselt er für den nächsten Frame sein Sendefrequenz auf eine der drei anderen ihm zugewiesenen Uplink-Spuren.

\section{Evaluierung:}

Bei einer Beleuchtungsstärke von $\mathrm{E}_{\mathrm{V}}=500 \mathrm{Ix}$ haben Messungen an einem Testsystem gezeigt, dass bei zwei Ereignissen pro Sekunde je Sensorknoten die Energiebilanz positiv ist, so dass keine Energie dem angeschlossenen Akku entnommen werden muss. Die Kapazität ist mit 100mAh auch groß genug, um bei einer Spitzenlast von $n=120$ Ereignissen pro Sekunde an einem Kommunikationsknoten den Betrieb über $\mathrm{t}=6 \mathrm{~h}$ zu ermöglichen. Es wurden bereits Sensorknoten mit je einem Endschalter oder einem resistiven Sensor zur Druck- bzw. Temperaturmessung getestet. Die maximale auftretende Latenzzeit auf der Funkschnittstelle $t_{\mathrm{Lmax}}$ berechnet sich aus der maximalen Anzahl $n_{\max }$ notwendiger Übertragungen und der Framedauer $t_{\mathrm{F}} \mathrm{zu}$

$$
t_{L \max }=n_{\max } \cdot t_{F}
$$

Damit liefert Gleichung 6 eine maximale Latenzzeit von $t_{\operatorname{Lmax}}=4 \mathrm{~ms}$, die mit einer Wahrscheinlichkeit von nur $10^{-9}$ überschritten wird.

Die obigen Überlegungen gehen von einer mittleren Kommunikationslast aus. Hält man während des Betriebs des Funksystems auch eine unvorhersehbare oder außerhalb des Modells des angenommenen Poisson-Prozesses liegende Häufung von Trigger-Ereignissen für möglich, stellt sich die Frage, wie schnell wird ein "Datenstau“ vom realisierten System behoben wird. Für die Beantwortung dieser Frage wurde getestet wie sich das System verhält wenn gleichzeitig 16 Sensorereignisse auftreten, alle 16 Kommunikationsknoten zu diesem Zeitpunkt auf einer Uplink-Spur senden und zudem die gleichen vier Uplink-Spuren dauerhaft nutzen. In Abbildung 7 ist das bei diesem Test gemessene Spektrogramm abgebildet. Es zeigt die Auslastung der zwei Downlink- und vier Uplink-Spuren. Der Zeitpunkt, an dem die 16 Kommunikationsmodule kollidieren, ist mit dem schwarzen Kreis gekennzeichnet. Nach diesem Zeitpunkt versuchen die 16 Kommunikationsknoten auf ihren vier Uplink-Spuren zu übertragen und erwarten auf den zwei Downlink-Spuren ihr ACKSignal. Innerhalb von $t_{F}=2,778 \mathrm{~ms}$ konnte der Datenstau durch die Routine zur Reduzierung der Kollisionswahrscheinlichkeit behoben werden. Damit wurde belegt, dass das System auch mit spontanen Ereignissen umgehen kann die wesentlich unwahrscheinlicher sind als die geforderte SER von $10^{-9}$. 


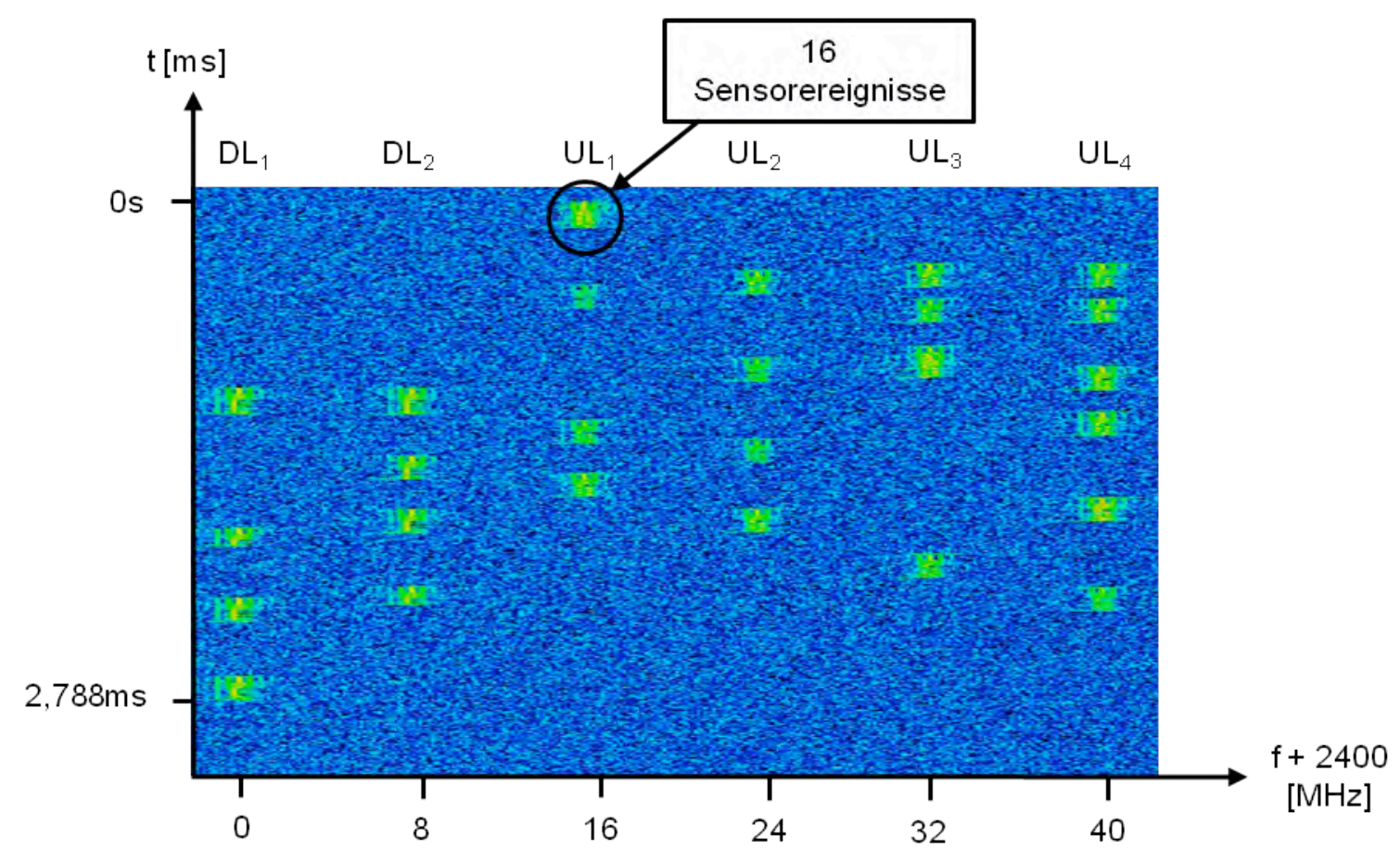

Abbildung 7: Spektrogramm des Worst-Case-Falls, 16 Sensorereignisse treten zum gleichen Zeitpunkt auf und werden innerhalb von $\mathrm{t}_{\mathrm{F}}=2,778 \mathrm{~ms}$ abgearbeitet.

In einem weiteren Test wurde speziell die Routine zur Reduzierung der Kollisionswahrscheinlichkeit untersucht. Dazu wurde acht Kommunikationsknoten nur eine Down- und eine Uplinkspur zugewiesen. Alle acht erhielten gleichzeitig ein Sensorereignis. Dies bedeutet für das gesamte System: von den 400 möglichen Sensoren müssten 64 innerhalb einer Zeitspanne von $25 \mu$ je ein Ereignis erhalten. Die Wahrscheinlichkeit hierfür liegt bei $4 \cdot 10^{-26}$. Die daraus resultierenden Kollisionen können hier nur durch das LBT-Verfahren in Verbindung mit dem zufälligen Versatz des Startzeitpunktes aufgelöst werden. Bei diesem Test konnte eine maximale Latenzzeit von 11,2 ms gemessen werden.

5. Zusammenfassung

Es wurde ein Funk-Kommunikationssystem für die Fertigungsautomatisierung vorgestellt, bei dem durch die geschickte Abstimmung von Hardware und MAC-Zugriffverfahren ein energieautarker Betrieb der Sensor-Kommunikationsmodule realisiert werden konnte. Bei einer Beleuchtungsstärke von $500 \mathrm{Ix}$ wurde von einem Solarpanel mit einer Fläche von $64 \mathrm{~cm}^{2}$ ausreichend Energie generiert, um im Mittel zwei Datenpakete pro Sekunde übertragen und die Acknowledgements von der Basisstation empfangen zu können. Mit der Auslegung der BS mit zehn parallel arbeitenden Standard HF-Transceivern und der Kommunikationsknoten mit jeweils einem Standard HF-Transceiver, der zudem nur bei Auftritt eines Sensorereignisses aus dem Sleep-Modus in den aktiven Modus übergeht, wurde einen unsymmetrischen Energiebedarf bei der Datenübertragung ermöglicht. Dieser wurde genutzt, um mit dem erweiterten CSMA-Verfahren ein Netzwerk mit 400 energieautarken Kommunikationsknoten zu realisieren. Dieses Netzwerk hat in ersten Messungen die von der Fertigungsautomatisierung geforderten kurzen Latenzzeiten und die hohe Zuverlässigkeit gezeigt.

Quellen:

[1] R. Heynicke, D. Krüger and G. Scholl, "Wireless Automation," Sensor + Test Conference, Nürnberg, Germany, June 2011.

[2] Enocean: Wireless sensor solutions for home \& building automation - The successful standard uses energy harvesting. White paper, Oberhaching 2007.

[3] J. Weczerek "Automatisierung funk(tioniert) mit Bluetooth und WLAN" A\&D Select Robotik \& Automation, pp. 22-25, January 2007.

[4] R. Heynicke, D. Krüger, H. Wattar, and G. Scholl, "Modular wireless fieldbus gateway for fast and reliable sensor/actuator communication," IEEE International Conference on Emerging Technologies and Factory Automation, Hamburg, Germany, pp. 1173-1176, September 2008. 
[5] R. Heynicke, D. Krüger, H. Wattar and G. Scholl, "Realisierung und Verifikation eines modularen drahtlosen Sensor/Aktor-Netzwerkes für die Fertigungsautomatisierung”, Automation 2009, Baden-Baden, Germany, June 2009.

[6] Paradiso, J.A.; Starner, T.: Energy Scavenging for Mobile and Wireless Electronics. IEEE Pervasive Computing, 4, S.18-27, 2005.

[7] Deutsches Institut für Normung: DIN EN 12464, Licht und Beleuchtung - Beleuchtung von Arbeitsstätten, Norm, Ausgabe August 2011.

[8] Linear Technology: LTC3105 400mA Step-Up DC/DC Converter with Maximum Power Point Control and 250mV Start-Up, Datenblatt, ver. A, 2010.

[9] Texas Instruments: MSP430F15x, MSP430F16x, MSP430F161x Mixed signal microcontroller, Datenblatt, Literaturnummer SLAS368G, März 2011.

[10] Texas Instruments: CC2400 2.4 GHz Low-Power RF Transceiver, Datenblatt, ver. 1.5, März 2006.

[11] H. Wattar, M. Thewes and G. Scholl "Modulares Messsystem zur Vermessung dynamischer Inhouse-Funkkanäle" Sensoren und Messsysteme, Nürnberg, Germany, May 2010.

[12] R. Heynicke, D. Krüger and G. Scholl, "Robuste, adaptive Wireless-Sensor/Aktor Kommunkation," in tm - Technisches Messen, Oldenbourg Wissenschaftsverlag, 2011, pp.196202.

[13] R. Heynicke, D. Krüger and G. Scholl, "Steigerung der Robustheit drahtloser Sensor/AktorFeldbussysteme durch prozessorientiertes adaptives Frequency-Hopping," Wireless technologies Congress, Bochum, Germany, September 2010.

[14] R. Heynicke, D. Krüger, H. Wattar and G. Scholl, "Robuste drahtlose Sensor/AktorKommunikation mit geringer Latenzzeit für die Fertigungsautomatisierung," XXIV Messtechnisches Symposium, Hamburg, Germany, September 2010.

[15] Abrahamson, N.: The Throughput of Packet Broadcasting Channels, IEEE Trans. on Com., vol. com-25, Nr. 1, Januar 1977.

[16] Kleinroch, L.: Packet Switching in Radio Channels: Part I-Carrier Sense Multiple-Access Modes and Their Throughput-Delay Characteristics, IEEE Trans. on Com., vol. com-23, Nr. 12, Dezember 1975. 